\title{
Long-term safety profile of tolvaptan in autosomal dominant polycystic kidney disease patients: TEMPO Extension Japan Trial
}

This article was published in the following Dove Press journal:

Drug, Healthcare and Patient Safety

25 October 2017

Number of times this article has been viewed

\author{
Satoru Muto' \\ Tadashi Okada² \\ Moriyoshi Yasuda ${ }^{3}$ \\ Hidetsugu Tsubouchi ${ }^{4}$ \\ Koji Nakajima ${ }^{4}$ \\ Shigeo Horie ${ }^{1,5}$ \\ 'Department of Advanced Informatics \\ for Genetic Disease, Juntendo \\ University Graduate School of \\ Medicine, Tokyo, ${ }^{2}$ Department \\ of Clinical Development, \\ ${ }^{3}$ Pharmacovigilance Department, \\ ${ }^{4}$ Department of Medical Affairs, \\ Otsuka Pharmaceutical Co, Ltd, \\ ${ }^{5}$ Department of Urology, Juntendo \\ University Graduate School of \\ Medicine, Tokyo, Japan
}

Aim: The aim of this trial (ClinicalTrials.gov identifier: NCT01280721) was to investigate the long-term safety profile of tolvaptan in Japanese patients with autosomal dominant polycystic kidney disease (ADPKD).

Methods: This open-label multicenter trial was conducted to examine adverse drug reactions (ADRs) related to tolvaptan up to an additional 3 years in 135 Japanese patients who participated in the Tolvaptan Efficacy and Safety in Management of Autosomal Dominant Polycystic Kidney Disease and its Outcomes (TEMPO) 3:4 trial at doses of 60-120 mg/d. Blood samples were collected at baseline; at weeks 1, 2, and 3; at month 3; and every 3 months thereafter. Results: In total, 134/135 (>99\%) patients experienced ADRs. The most frequent ADRs were thirst $(77.0 \%)$, pollakiuria $(57.0 \%)$, polyuria (37.8\%), and hyperuricemia (14.8\%). Any unexpected ADRs were not reported in this trial. Most ADRs occurred early during treatment. Fourteen patients (10.4\%) experienced hepatic events, and 8 (5.9\%) experienced $>3$-fold increases above the upper limits of normal in serum alanine aminotransferase or aspartate aminotransferase levels between 3 and 9 months following tolvaptan initiation, which recovered after drug interruption. Of the 8 patients, 7 (5.2\%) were previously allocated to the placebo arm in the TEMPO 3:4 trial and $4(3.0 \%)$ discontinued due to the hepatic events. One patient $(0.7 \%)$ was previously allocated to tolvaptan and experienced similar events in the TEMPO 3:4 trial. None of the hepatic ADRs met Hy's Law laboratory criteria.

Conclusion: ADRs observed in this extension trial were similar to those identified in the TEMPO 3:4 trial and hepatic events were not progressive.

Keywords: autosomal dominant polycystic kidney disease, drug-induced liver injury, liver function test, safety profile, tolvaptan

\section{Introduction}

Autosomal dominant polycystic kidney disease (ADPKD) is an inherited disease associated with progressive increase in total kidney volume (TKV), leading to deterioration of kidney function, and almost $50 \%$ of patients reach end-stage renal disease. ${ }^{1-3}$ Patients with ADPKD maintain a glomerular filtration rate in the normal range for decades. However, continuous cyst growth and proliferation results in loss of kidney function over time, and the estimated glomerular filtration rate (eGFR) only starts to decline after the kidneys are grossly enlarged and little normal renal parenchyma remains.

Early in vivo studies demonstrated that tolvaptan, a potent, highly selective, and orally effective nonpeptide arginine vasopressin $\mathrm{V}_{2}$ receptor antagonist, reduced kidney cyclic adenosine $3^{\prime}, 5^{\prime}$-monophosphate levels and kinase activity, in addition to slowing disease progression. ${ }^{4-6}$ In the pivotal Tolvaptan Efficacy and Safety in Management
Correspondence: Satoru Muto Department of Advanced Informatics for Genetic Disease, Juntendo University Graduate School of Medicine, Hongo 2-I-I, Bunkyo-ku, Tokyo II 3-842I, Japan $\mathrm{Tel}+8 \mathrm{I} 35802$ I227

Fax +8I $35803 \quad 227$

Email s-muto@juntendo.ac.jp 
of Autosomal Dominant Polycystic Kidney Disease and Its Outcomes (TEMPO 3:4) trial (ClinicalTrials.gov identifier: NCT00428948), tolvaptan slowed the annual rate of increase in TKV and slowed the decline in kidney function. ${ }^{7,8}$ Tolvaptan was approved for the treatment of ADPKD in Japan (March 2014), Canada (February 2015), the European Union (May 2015), Korea (December 2015), Switzerland (April 2016), and Hong Kong (April 2017).

In the Japanese subset from TEMPO 3:4 ( $n=118), 3$ patients experienced an abnormality in hepatic function classed as serious adverse events (SAEs), and all recovered following drug interruption. Of the 3 patients, 1 met Hy's Law laboratory criteria, ${ }^{9}$ defined as $3 \times$ the upper limit of normal range (ULN) in serum alanine aminotransferase (ALT) or aspartate aminotransferase (AST) levels and $2 \times$ the ULN in serum total bilirubin (T-Bil), defined according to Guidance for Industry: Drug-Induced Liver Injury (DILI). ${ }^{9}$ The manifestation of hepatocellular injury following long-term tolvaptan treatment in ADPKD revealed the potential for serious irreversible injury; thus, we designed an open-label trial to evaluate the safety profile of tolvaptan in Japanese ADPKD patients following completion of the TEMPO 3:4 trial. In the meantime, the open-label extension trial TEMPO 4:4 (ClinicalTrials.gov identifier: CT01214421, 2010-018401-10) was designed to provide an additional 2 years of data on the long-term safety and efficacy of tolvaptan in subjects who completed TEMPO $3: 4$, and this was conducted outside of Japan. ${ }^{10}$

\section{Methods}

\section{Open-label trial design}

This open-label, multicenter, Phase III trial (TEMPO Extension Japan trial) was performed at 30 trial sites in Japan from November 2010 through August 2014. It was conducted in accordance with ethical principles originating from the
Declaration of Helsinki and in compliance with good clinical practice guidelines. The protocol was approved by the institutional review board (IRB) at each trial site (Table S1). The first IRB approval was issued by Niigata University (IRB approval number: $\mathrm{CH} 22-010)$. All patients provided written informed consent. Tolvaptan was administered to Japanese patients who completed the TEMPO 3:4 trial until its approval for the indication of ADPKD in Japan.

This trial consisted of a screening period ( 5 weeks), a titration period ( 3 weeks), an additional treatment period, the final evaluation period ( 1 week), and the follow-up period (8 weeks) (Figure 1). Patients who provided written informed consent underwent the screening evaluation, including vital sign tests, TKV measurements, clinical laboratory tests, electrocardiogram examinations, and kidney function tests.

The inclusion criteria were as follows:

1. Japanese patients who completed the TEMPO 3:4 trial with completed case report forms;

2. Patients who either completed 3 years of repeated administration in the TEMPO 3:4 trial or who discontinued due to pregnancy, and who completed the second follow-up examination in that trial;

3. Patients who had stable adverse events (AEs) or recovered from AEs in the TEMPO 3:4 trial and did not require follow-up examination.

The exclusion criteria were as follows:

1. Patients with eGFR $<15 \mathrm{~mL} / \mathrm{min} / 1.73 \mathrm{~m}^{2}$;

2. Patients who received trial drugs other than tolvaptan within 30 days before initiation of tolvaptan use;

3. Female patients who were pregnant, breastfeeding, or suspected to be pregnant, or those who intended to become pregnant during this trial;

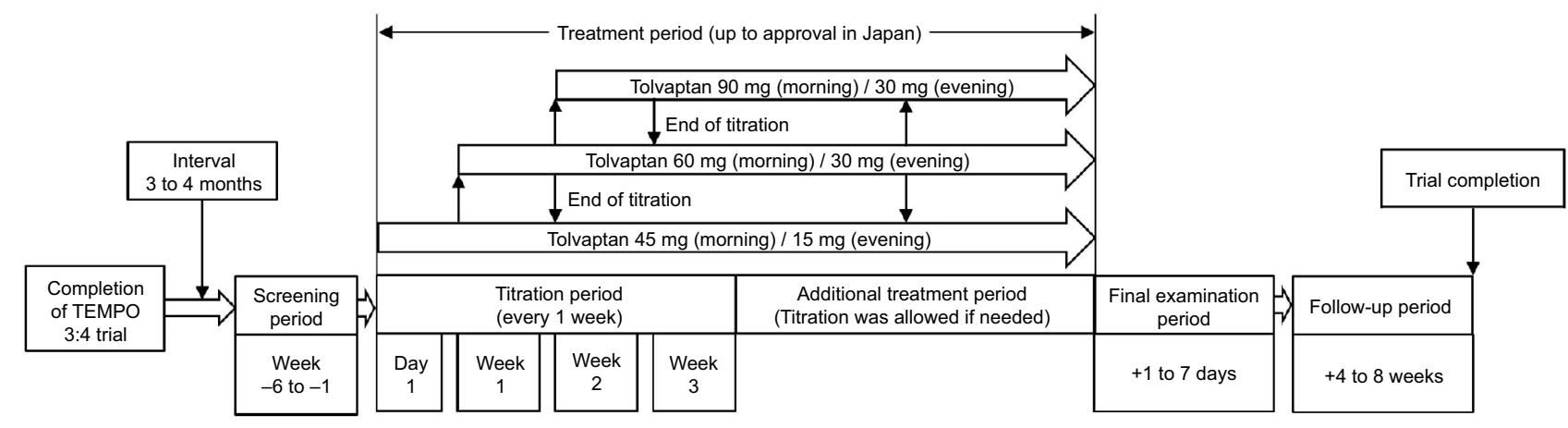

Figure I Design of TEMPO Extension Japan Trial.

Notes: This trial involved patients who were previously enrolled in the TEMPO 3:4 trial. ${ }^{7.8}$ All participants received tolvaptan. Titration method was according to the previous TEMPO 3:4 trial.

Abbreviation: TEMPO, Tolvaptan Efficacy and Safety in Management of Autosomal Dominant Polycystic Kidney Disease and its Outcomes. 
4. Any patient who, in the opinion of the investigator or subinvestigator, should not participate in the trial.

Included patients underwent the baseline evaluation to reconfirm their eligibility immediately before initiation of tolvaptan (baseline data). Tolvaptan was administered to patients in a split-dose regimen in the morning and in the evening during both the titration period and the additional treatment period until tolvaptan was approved for treatment of ADPKD in Japan. Thus, an available maximum amount of time until the approval of tolvaptan was set as the trial period for each patient to obtain longer-term safety data. Three split-dose regimens of oral tolvaptan were tested for patients: a low dose of $60 \mathrm{mg} / \mathrm{d}(45 / 15 \mathrm{mg} / \mathrm{d})$, an intermediate dose of $90 \mathrm{mg} / \mathrm{d}(60 / 30 \mathrm{mg} / \mathrm{d})$, and a high dose of $120 \mathrm{mg} / \mathrm{d}$ $(90 / 30 \mathrm{mg} / \mathrm{d})$. In the 3 -week titration period, patients began treatment with the lowest dose of tolvaptan, and doses were titrated to the next highest doses after each 1-week confirmation of a patients' self-reported tolerability. If higher doses were not well tolerated, downtitration was allowed. Thus, the optimal dose of tolvaptan according to tolerability was decided for each patient.

Following the titration period, patients remained on the highest tolerable doses during the additional treatment period. However, uptitration or downtitration was permitted according to patients' tolerability and/or clinician's decision within the 3 defined dosing regimens.

The final evaluation was conducted for patients within 7 days after the final administration of tolvaptan. The follow-up evaluation was conducted within 4-8 weeks after the final administration. If patients discontinued tolvaptan treatment for any reason, the same evaluation as the final one was performed (the discontinuation evaluation). Patients who received at least 1 dose of tolvaptan and had at least 1 observation after initiation of tolvaptan were included in the safety assessment.

\section{Safety assessment}

Safety assessment included documentation of adverse drug reactions (ADRs), clinical laboratory tests, vital signs, and electrocardiogram recordings.

Data on ADRs, which depended on physician judgment, were collected throughout the trial. The time points of data collection after initiation of tolvaptan use were as follows. Data on clinical laboratory test results were collected at baseline; at weeks 1, 2, and 3; at month 3; and every 3 months thereafter. Data on vital sign results were collected at baseline at weeks 1, 2, 3, and 4; and each month thereafter. Data on electrocardiogram examinations were collected at baseline, at week 4, at month 12 , and every 12 months thereafter. These data were also collected at the final or discontinuation evaluations, as well as at the follow-up evaluation. The discontinuation or interruption criteria for individual patients were as follows:

1. Withdrawal of consent;

2. Deterioration of renal function (eGFR value $<15 \mathrm{~mL} /$ $\left.\min / 1.73 \mathrm{~m}^{2}\right)$;

3. Detection of hepatocellular injury (ALT or AST: $>3 \times$ ULN and T-Bil: $>2 \times$ ULN);

4. Inability of patient to tolerate the lowest dose regimen (45/15 mg);

5. Patients for whom protocol compliance becomes impossible or for whom the principal investigator or attending subinvestigators judge withdrawal to be necessary for reasons other than those described earlier.

In addition, when any of the biochemical findings listed herein was detected, treatment was suspended promptly (refer to the US Food and Drug Administration [FDA] Guidance for Industry: Drug-induced Liver Injury, 2009).

1. ALT or AST $>8 \times$ ULN;

2. ALT or AST $>5 \times$ ULN, which persists for $>2$ weeks;

3. AST or AST $>3 \times$ ULN and T-Bil $>2 \times$ ULN or prothrombin time (international normalized ratio [INR]) $>1.5$;

4. ALT or AST level greater than 3 times the ULN with appearance of fatigue, nausea, vomiting, right upper quadrant pain or tenderness, fever, rash, and/or eosinophilia $(>5 \%)$.

ADRs, together with their severities and occurrence time points, were observed after initiation of tolvaptan use. Severity of ADRs was defined as mild when uncomfortable feelings were experienced without difficulty in daily living activity, as moderate when uncomfortable feeling was experienced with limitation or negative influence on daily living activity, and as severe when disability in daily living activity or work occurred. For recurring events in the same patient, the first observation time point was analyzed.

Clinical laboratory tests for measuring serum sodium and potassium levels, as well as serum ALT, AST, and T-Bil levels as biomarkers of hepatic functions, were conducted at each time point.

\section{Statistical analysis}

No formal hypothesis testing was performed in the open-label trial. Continuous variables were expressed as a mean value 
with SD. Categorical variables were expressed as number and proportion. These data were calculated using SAS 9.2 (SAS Institute, Cary, NC, USA).

\section{Results}

\section{Demographic characteristics of patients and dose of tolvaptan}

In the TEMPO Extension Japan trial, 135 patients were eligible to initiate treatment with tolvaptan and were included in the safety assessment. No patients were excluded from this trial to analyze the safety profile of tolvaptan. Demographic and clinical characteristics of patients at baseline are described in Table 1. During the long-term treatment period, the mean dose of tolvaptan was $91.7 \pm 25.7 \mathrm{mg} / \mathrm{d}$, with the majority of patients (47.7\%) being able to tolerate the highest dose of $120 \mathrm{mg} / \mathrm{d}$ $(\mathrm{n}=63)$. Remaining patients were on $90 \mathrm{mg} / \mathrm{d}(\mathrm{n}=27,20.5 \%)$ and $60 \mathrm{mg} / \mathrm{d}(\mathrm{n}=42,31.8 \%)$. The mean duration of tolvaptan administration was $856.3 \pm 299.8$ days (range: $7-1264$ days).

\section{Safety assessment}

Patient flow from the TEMPO 3:4 trial to the TEMPO Extension Japan trial is shown in Figure 2. Totally, 12 patients, 7 of tolvaptan and 5 of placebo, did not participate in the extension trial. In total, 22 patients $(16.3 \%)$ discontinued this trial, 4 (3.0\%) due to AEs, 9 (6.7\%) due to meeting discontinuation criteria, $3(2.2 \%)$ due to discretion by clinicians, and $6(4.4 \%)$ due to withdrawal of consent. The remaining 113 (83.7\%) patients completed the treatment period up to approval. In

Table I Demographic and clinical characteristics at baseline

\begin{tabular}{ll}
\hline Characteristics & $\mathbf{n}=135$ \\
\hline Age, years & $42.7 \pm 5.9$ \\
Sex, $\mathrm{n}(\%)$ & $77(57.0)$ \\
$\quad$ Male & $58(43.0)$ \\
$\quad$ Female & $168.3 \pm 8.1$ \\
Height, $\mathrm{cm}$ & $67.6 \pm 13.3$ \\
Body weight, $\mathrm{kg}$ & $23.7 \pm 3.5$ \\
Body mass index & $1812 \pm 859$ \\
TKV, $\mathrm{mL}$ & $1073 \pm 493$ \\
htTKV, $\mathrm{mL} / \mathrm{m}$ & $61.2 \pm 21.7$ \\
eGFR, $\mathrm{mL} / \mathrm{min} / \mathrm{I.73} \mathrm{m}^{2}$ & $1.11 \pm 0.55$ \\
Serum creatinine, $\mathrm{mg} / \mathrm{dL}$ & $1.02 \pm 0.42$ \\
Serum cystatin C, $\mathrm{mg} / \mathrm{L}$ & $19.9 \pm 16.9$ \\
Serum ALT, U/L & $22.2 \pm 9.7$ \\
Serum AST, U/L & $0.60 \pm 0.22$ \\
Total bilirubin, $\mathrm{mg} / \mathrm{dL}$ & $140.1 \pm 1.7$ \\
Serum sodium, $\mathrm{mEq} / \mathrm{L}$ & $4.09 \pm 0.33$ \\
Serum potassium, $\mathrm{mEq} / \mathrm{L}$ &
\end{tabular}

Note: Data are expressed as number (\%) or mean \pm SD.

Abbreviations: ALT, alanine aminotransferase; AST, aspartate aminotransferase; eGFR, estimated glomerular filtration rate; htTKV, height-corrected total kidney volume; TKV, total kidney volume. total, 512 ADRs were reported from 134 patients. Among them, 9 serious ADRs from 4 patients, and 16 severe ADRs from 8 patients were reported. Moreover, 6 patients discontinued tolvaptan use due to ADRs. The most frequent ADR was thirst $(77.0 \%)$, followed by pollakiuria $(57.0 \%)$, polyuria (37.8\%), hyperuricemia (14.8\%), and hepatic events (10.4\%), which were all observed at rates of $>10 \%$ of included patients (Table 2). AEs of cardiac disorder were reported from 13 patients (9.6\%) and ADRs were found in 6 patients (4.4\%). New dialysis cases were not reported in this study.

ADRs were generally mild in severity and were observed in the first 3 months of treatment (Table 2). Occurrence rates of the first ADRs in patients were $97.8 \%$ within 3 months, $0.7 \%$ in 3-6 months, $0 \%$ in 6-9 months, $0 \%$ in $9-12$ months, and $0 \%$ in $12-30$ months (Figure 3). The main ADRs observed after 3 months of treatment were hepatic function abnormality and hyperuricemia.

A total of 9 serious ADRs were found in 4 patients, namely, renal cyst infection (twice in 1 patient), sepsis $(n=1)$, ovarian cancer $(n=1)$, uterine cancer $(n=1)$, dizziness $(n=1)$, renal cyst hemorrhage $(n=1)$, renal cyst rupture $(n=1)$, and renal pain $(n=1)$. All 4 patients were hospitalized due to the events.

Time course profiles of mean serum ALT, AST, and T-Bil are presented in Figure 4. Fourteen patients showed hepatic events. Of those, 8 patients (5.9\%) experienced a $>3$-fold increase above the ULN in serum ALT or AST levels between 3 and 9 months after initiation of tolvaptan use, but 6 of those recovered after interruption of tolvaptan and 2 recovered without interruption (Table 3). Of the 8 patients, 5 discontinued the trial, and 3 completed the trial by continuation of tolvaptan or restarted after interruption. None of the hepatic events met Hy's Law laboratory criteria. Eight patients (5.9\%) experienced an increase in blood creatinine levels (7 mild and 1 moderate in severity), which was judged by clinicians as an ADR (Table 2). In 6 of these patients, blood creatinine increased within 15 days after initiation of tolvaptan use. Six patients recovered without dose adjustment and 1 patient with moderate severity recovered following downtitration of tolvaptan dose, while 1 patient discontinued. Time courses of mean serum sodium and potassium levels during treatment are presented in Figure 5. No other deterioration of laboratory tests was observed. No marked abnormalities were observed in vital signs and electrocardiogram recordings. No death was observed.

\section{Discussion}

We focused on evaluating the safety profile of tolvaptan in the Japanese subset of ADPKD patients from the TEMPO 3:4 


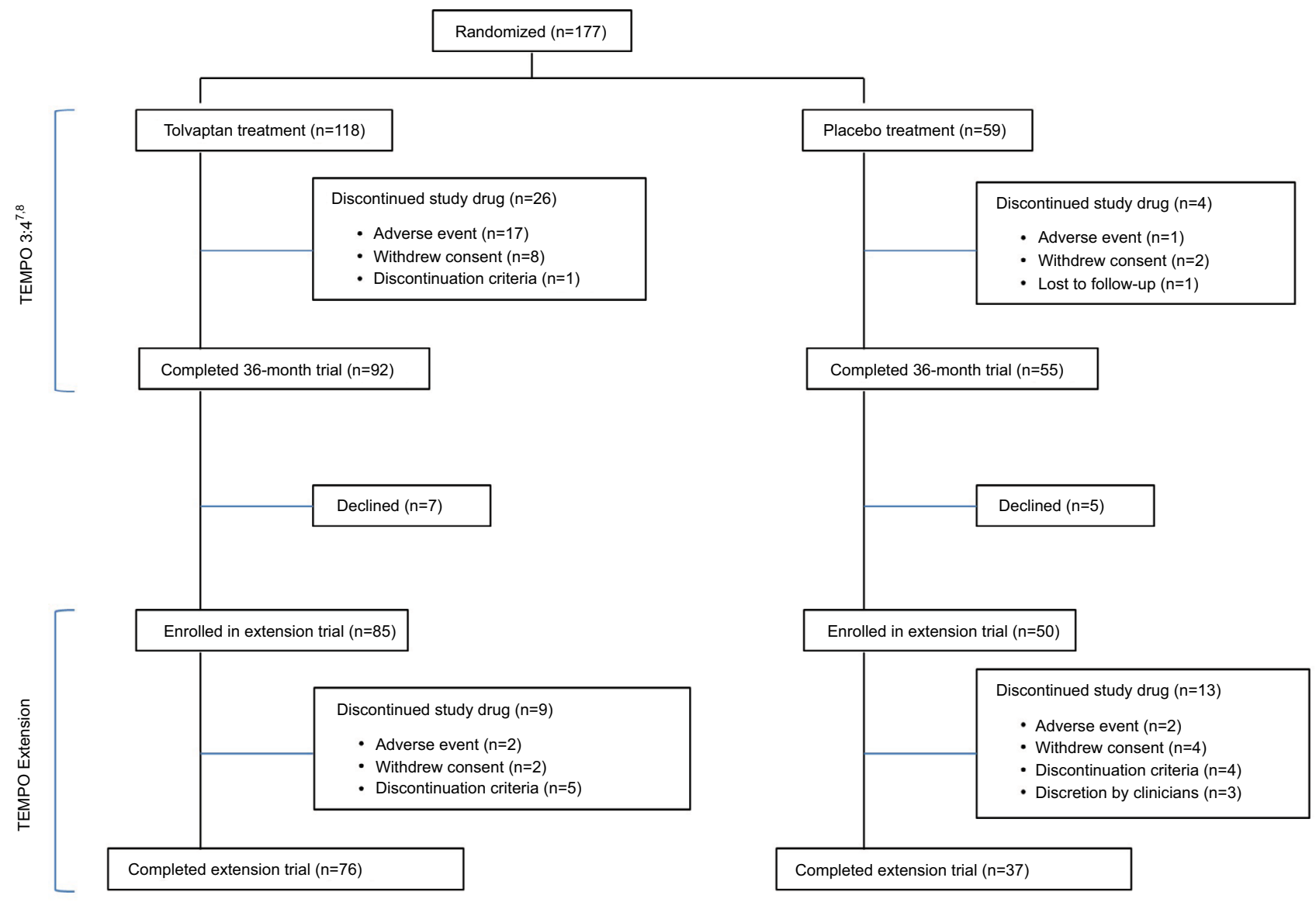

Figure 2 Patient flow chart.

Abbreviation: TEMPO, Tolvaptan Efficacy and Safety in Management of Autosomal Dominant Polycystic Kidney Disease and its Outcomes.

trial through to marketing approval in Japan. ADRs observed in this extension trial were similar to those reported in Japanese patients in the TEMPO 3:4 trial. ${ }^{8}$ Most ADRs occurred acutely within 3 months following initiation of tolvaptan use, and the severity of ADRs was generally mild. In the present trial, we stratified the population according to the treatment arm in the pivotal TEMPO 3:4 trial in Japan and calculated withdrawal rates in each group (Figure 6). In the TEMPO $3: 4$ trial, 4 placebo patients (6.8\%) and 26 tolvaptan patients (22.0\%) did not complete the trial. Similarly, placebo patients who crossed over onto active treatment discontinued at a similar rate $(24.0 \%)$, while those who continued on tolvaptan treatment discontinued at a much lower rate (11.8\%). Moreover, the percentage of patients reporting a hepatic event was similar across both studies $(9.6 \%$ in the open-label trial vs $10.2 \%$ in the TEMPO $3: 4$ trial). ${ }^{8}$

ADRs that occurred in $\geq 10 \%$ of patients were thirst, pollakiuria, polyuria, hyperuricemia, and hepatic events. With the exception of the hepatic events, other ADRs were consistent with the pharmacological effect of tolvaptan. ${ }^{6,12}$
Serious ADRs observed in this trial included sepsis, renal cyst infection, ovarian cancer, uterine cancer, dizziness, renal cyst hemorrhage, renal cyst rupture, and renal pain. Serious ADRs such as renal cyst infection, renal cyst hemorrhage, renal cyst rupture, and renal pain are common manifestations of ADPKD.

In the TEMPO 3:4 trial, 1 Japanese patient in the tolvaptan arm met Hy's Law laboratory criteria, resulting in discontinuation from the trial. ${ }^{7}$ In the present trial, hepatic events were marked ADRs found at a rate of $\sim 10 \%$. Eight patients showed ALT and AST elevations $>3$-fold the ULN between 3 and 9 months following treatment initiation. One of these patients had received tolvaptan in the TEMPO 3:4 trial. However, this patient initially had high baseline levels of ALT and AST in the present trial. Moreover, this patient eventually discontinued the trial due to renal impairment and not due to a hepatic event; the remaining 7 patients (prior placebo patients in the TEMPO 3:4 trial) had normal hepatic function values at baseline of the extension trial. Of the 7 patients, 4 discontinued the trial due to their hepatic events and the 
Table 2 Adverse drug reactions observed in patients treated with tolvaptan and their severity and observed time points

\begin{tabular}{|c|c|c|c|c|c|c|c|c|c|}
\hline \multirow[t]{2}{*}{ Events } & \multirow[t]{2}{*}{ n (\%) } & \multicolumn{3}{|c|}{ Severity } & \multicolumn{5}{|c|}{$\begin{array}{l}\text { Observed time point after initiation of } \\
\text { tolvaptan use, months }\end{array}$} \\
\hline & & Mild & Moderate & Severe & $\leq 3$ & to $\leq 6$ & to $\leq 9$ & to $\leq 12$ & to $\leq 30$ \\
\hline \multicolumn{10}{|l|}{ State of general disorders } \\
\hline Thirst & $104(77.0)$ & 91 & 12 & I & 103 & - & - & - & I \\
\hline Hepatic events & $14(10.4)$ & - & - & - & - & - & - & - & - \\
\hline Drug-induced liver injury & I (0.7) & I & - & - & 1 & - & - & - & - \\
\hline Abnormal hepatic function & $13(9.6)$ & 12 & I & - & 4 & 6 & 2 & - & I \\
\hline \multicolumn{10}{|l|}{ Clinical examination } \\
\hline Blood creatinine increase & $8(5.9)$ & 7 & I & - & 6 & - & I & - & I \\
\hline \multicolumn{10}{|c|}{ Metabolism and nutritional status } \\
\hline Decreased appetite & $5(3.7)$ & 3 & 2 & - & 4 & I & - & - & 0 \\
\hline Dehydration & $3(2.2)$ & 3 & - & - & 3 & - & - & - & - \\
\hline Hyperuricemia & $20(14.8)$ & 20 & - & - & 9 & 3 & I & - & 7 \\
\hline \multicolumn{10}{|l|}{ Nervous system disorders } \\
\hline Headache & $10(7.4)$ & 8 & 2 & - & 7 & - & 1 & - & 2 \\
\hline Mental disorder & - & - & - & - & - & - & - & - & - \\
\hline Insomnia & $6(4.4)$ & 2 & 4 & - & 4 & - & - & - & 2 \\
\hline \multicolumn{10}{|c|}{ Kidney and urinary tract disorders } \\
\hline Nocturia & $6(4.4)$ & 2 & 4 & - & 5 & 1 & - & - & - \\
\hline Pollakiuria & $77(57.0)$ & 52 & 24 & I & 75 & 2 & - & - & - \\
\hline Polyuria & $51(37.8)$ & 39 & 12 & - & 51 & - & - & - & - \\
\hline Renal impairment & $5(3.7)$ & 5 & - & - & 2 & - & I & - & 2 \\
\hline Vascular disorders & - & - & - & - & - & - & - & - & - \\
\hline Hypertension & $6(4.4)$ & 5 & I & - & 3 & - & I & I & 1 \\
\hline Hypotension & $4(3.0)$ & 4 & - & - & 2 & 1 & - & - & 1 \\
\hline
\end{tabular}

Notes: Predictable ADRs, such as liver function abnormality, and those from the aquaretic action of tolvaptan, including kidney function disorders, in addition to vascular events, are listed. As for the ADRs, the time point of the first ADR observed was analyzed. Events were categorized according to the MedDRA. Blank columns denote no ADRs. Data are expressed as the number of patients experiencing the respective ADRs, and the figures in parentheses demonstrate the percentage of those among the total patients who were administered the test drug $(n=135)$.

Abbreviations: ADR, adverse drug reaction; MedDRA, Medical Dictionary for Regulatory Activities.

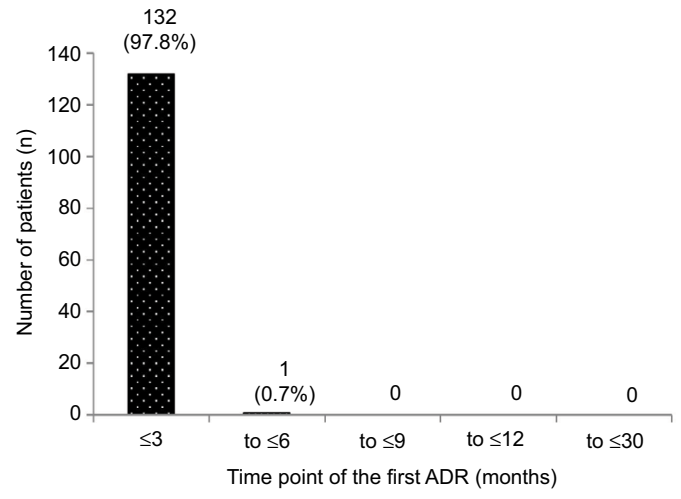

Figure 3 The number of patients experiencing their first ADRs in the course of the trial.

Notes: In the respective patients, the time point of the first ADR observed was noted. Thereafter, the numbers of the patients experiencing first ADR were analyzed every 3 months.

Abbreviation: ADR, adverse drug reaction.

remaining 3 completed the trial either continuing the drug or on treatment following drug interruption (Figure 6). None of the patients met Hy's Law laboratory criteria. Therefore, it is possible that monthly liver enzyme monitoring was sufficient to identify patients early in the hepatocellular injury event, thus preventing those patients from reaching levels consistent with Hy's Law laboratory criteria. All hepatic events observed in this trial were reversible; 2 patients recovered while on treatment (Patients 6 and 7 in Table 3). The 4 patients who discontinued due to hepatic function abnormalities were previously allocated to the placebo arm in the TEMPO 3:4 trial. All liver enzyme elevations occurred from 3 to 14 months following initiation of tolvaptan treatment, which is consistent with the 18-month window of susceptibility identified in the TEMPO 3:4 trial. ${ }^{8,11}$ The potential for serious irreversible injury exists; thus, regular monitoring of transaminase levels will be warranted in this patient population to avoid serious deterioration of hepatic function over long-term treatment with tolvaptan. In the TEMPO 3:4 trial and the interim analysis of TEMPO 4:4, the risk of liver failure in ADPKD patients receiving long-term tolvaptan was estimated to be approximately 1:4000. ${ }^{11}$ In Japan, tolvaptan was approved for the treatment of ADPKD on the condition that patients should receive monthly hepatic monitoring, a requirement that has been mirrored in other regions where marketing authorization has been granted. The contributing factor to hepatic injury is suggested to be the inhibition of hepatic bile 
A

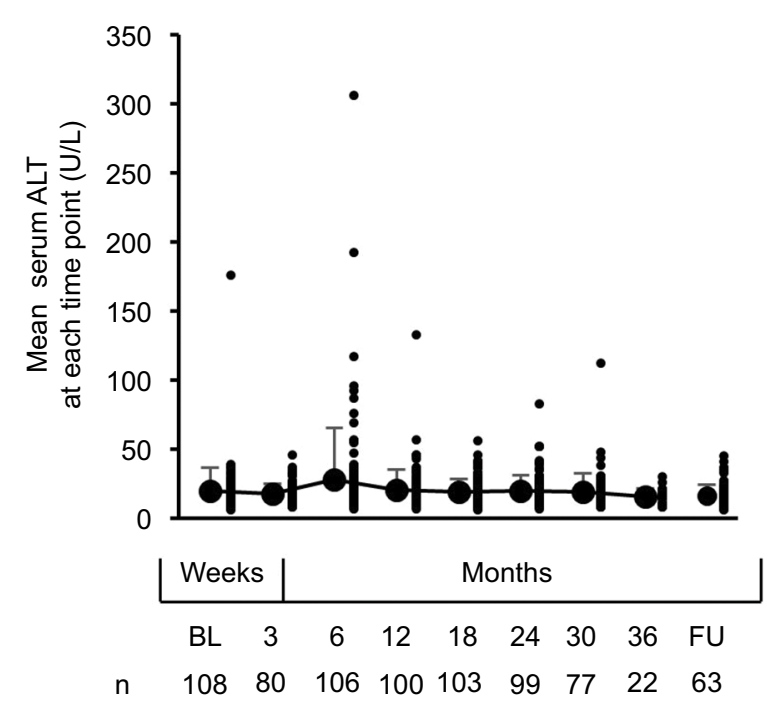

B

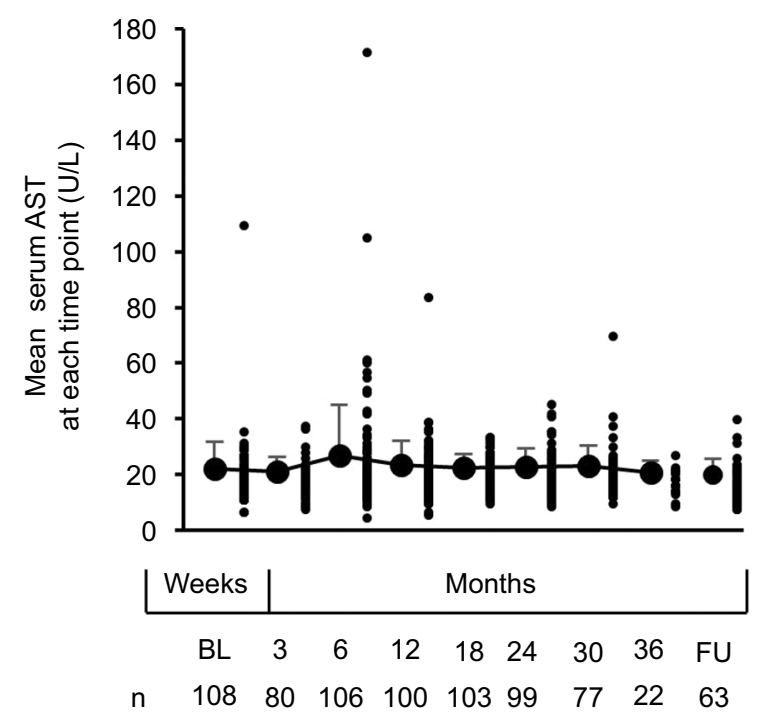

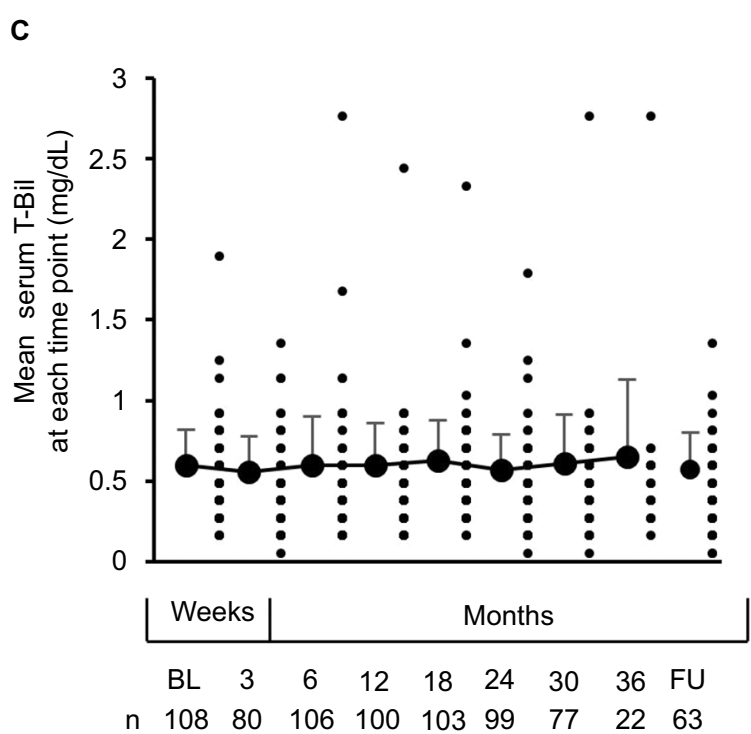

Figure 4 Laboratory test results at each time point.

Notes: (A) Serum ALT, (B) serum AST, and (C) serum T-Bil levels. Data are expressed as mean \pm SD. Ranges for normal values are as follows: ALT, 5-45 U/L; AST, I0-40 U/L; T-Bil, 0.2-1.2 mg/dL.

Abbreviations: ALT, alanine aminotransferase; AST, aspartate aminotransferase; T-Bil, total bilirubin; BL, baseline; FU, follow-up; n, number of patients.

acid transporters by tolvaptan and its metabolites. ${ }^{12}$ Further investigation of the mechanism of tolvaptan-induced liver injury might contribute to better clinical management.

On the other hand, 8 patients experienced increase in blood creatinine. Although there were similar reports, it is not clear yet whether tolvaptan is the single cause for this. ${ }^{7,8}$ However, in this study, the creatinine increase was likely to be transient, and 7 patients continued tolvaptan use, except for 1 discontinuation, resulting in recovery.

Hypernatremia is also a predictable ADR for tolvaptan because of its aquaretic action. According to the postmarketing surveillance for heart failure patients with volume overload, ${ }^{13}$ hypernatremia has been observed almost within 1 week after tolvaptan initiation. In this trial, no patient showed hypernatremia as an ADR. One of the reasons could be that water intake was not restricted in this trial, although it was commonly set in other trials to prevent edema. The limitation of this trial in terms of the study design was that it was not a comparative trial; moreover, efficacy analysis of tolvaptan was not performed. In this trial, we effectively conducted the treatment until approval of tolvaptan to obtain information on the long-term safety profile in an open-label manner in patients included in the TEMPO 3:4 trial. In the meantime, the open-label extension trial TEMPO 4:4 was conducted outside of Japan. ${ }^{10}$ Of the 1445 patients ( $n=961$ 
Table 3 Laboratory test results over time in patients showing an increase in serum ALT or AST to $>3$ times the upper limit of normal

\begin{tabular}{|c|c|c|c|c|c|c|c|c|c|c|c|c|c|c|c|c|c|c|c|c|}
\hline \multirow{2}{*}{$\begin{array}{l}\text { Patient } \\
\text { number }\end{array}$} & \multirow[t]{2}{*}{ Parameter } & \multirow[t]{2}{*}{ Baseline } & \multicolumn{16}{|c|}{ Month } & \multirow{2}{*}{$\begin{array}{l}\text { Dose, }{ }^{a} \\
\text { mg }\end{array}$} & \multirow{2}{*}{$\begin{array}{l}\text { Allocation in } \\
\text { TEMPO 3:4 } \\
\text { trial }\end{array}$} \\
\hline & & & 3 & 4 & 5 & 6 & 7 & 8 & 9 & II & 12 & 17 & 18 & 20 & 24 & 30 & 33 & 35 & & \\
\hline \multirow[t]{3}{*}{ I } & ALT & 176 & 92 & - & - & 87 & - & $177^{b}$ & 239 & 169 & 133 & 47 & 46 & 79 & 83 & - & - & - & 60 & Tolvaptan \\
\hline & AST & 107 & 55 & - & - & 44 & - & $132^{\mathrm{b}}$ & 177 & 103 & 83 & 31 & 27 & 52 & 47 & - & - & - & & \\
\hline & & - & - & - & - & - & - & - & $\star$ & - & - & - & - & - & $\mathbf{\Delta}^{c}$ & - & - & - & & \\
\hline \multirow[t]{3}{*}{2} & ALT & 18 & 426 & 173 & 300 & 35 & 19 & - & - & - & - & - & - & - & - & - & - & - & 60 & Placebo \\
\hline & AST & 21 & 175 & 87 & 147 & 29 & 21 & - & - & - & - & - & - & - & - & - & - & - & & \\
\hline & & - & $\star$ & - & - & - & $\boldsymbol{\Delta}$ & - & - & - & - & - & - & - & - & - & - & - & & \\
\hline \multirow[t]{3}{*}{3} & ALT & 17 & 236 & 392 & 221 & 65 & $21^{b}$ & 70 & 33 & - & - & - & - & - & - & - & - & - & 120 & Placebo \\
\hline & AST & 19 & 72 & 178 & 72 & 34 & $21^{b}$ & 45 & 29 & - & - & - & - & - & - & - & - & - & 60 & \\
\hline & & - & $\star$ & - & - & - & is & $\star$ & $\boldsymbol{\Delta}$ & - & - & - & - & - & - & - & - & - & & \\
\hline \multirow[t]{3}{*}{4} & ALT & 20 & 152 & - & 86 & 192 & 22 & 15 & 15 & 18 & 17 & 19 & 56 & 20 & - & - & - & - & 90 & Placebo \\
\hline & AST & 20 & 66 & - & 59 & 103 & 22 & 16 & 18 & 19 & 18 & 20 & 36 & 19 & - & - & - & - & 60 & \\
\hline & & - & $\star$ & - & - & - & - & - & - & - & - & $i$ & $\star$ & $\Delta$ & - & - & - & - & & \\
\hline \multirow[t]{3}{*}{5} & ALT & 20 & 18 & - & - & 76 & 691 & 241 & 78 & 20 & 26 & - & 16 & - & 11 & 12 & 10 & - & 60 & Placebo \\
\hline & AST & 25 & 25 & - & - & 58 & 439 & 179 & 61 & 27 & 30 & - & 23 & - & 21 & 20 & 20 & - & & \\
\hline & & - & - & - & - & - & $\star$ & - & - & is & - & - & - & - & - & - & () & - & & \\
\hline \multirow[t]{3}{*}{6} & ALT & 21 & 24 & 164 & 47 & 24 & - & - & 23 & - & 19 & - & - & - & 14 & 16 & 17 & - & 90 & Placebo \\
\hline & AST & 32 & 32 & 92 & 38 & 31 & - & - & 28 & - & 26 & - & - & - & 24 & 24 & 24 & - & & \\
\hline & & - & - & - & - & - & - & - & - & - & - & - & - & - & - & - & ()) & - & & \\
\hline \multirow[t]{3}{*}{7} & ALT & 17 & 18 & - & - & 117 & $180^{\mathrm{b}}$ & $121^{\mathrm{b}}$ & 238 & 59 & 59 & - & 30 & - & 28 & 22 & 19 & 19 & 60 & Placebo \\
\hline & AST & 17 & 21 & - & - & 61 & $75^{b}$ & $56^{\mathrm{b}}$ & 108 & 43 & 39 & - & 29 & - & 25 & 22 & 23 & 23 & & \\
\hline & & - & - & - & - & - & - & - & - & - & - & - & - & - & - & - & - & (0) & & \\
\hline \multirow[t]{3}{*}{8} & ALT & 18 & 96 & 147 & 522 & 306 & 37 & 18 & 16 & - & - & - & - & - & - & - & - & - & 120 & Placebo \\
\hline & AST & 25 & 70 & 92 & 261 & 165 & 31 & 26 & 22 & - & - & - & - & - & - & - & - & - & & \\
\hline & & - & $\star$ & - & - & - & - & - & $\boldsymbol{\Delta}$ & - & - & - & - & - & - & - & - & - & & \\
\hline
\end{tabular}

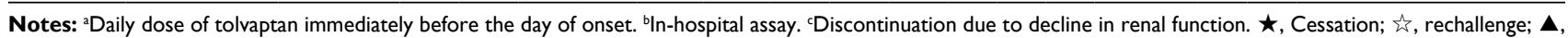
discontinuation; (), completion. Normal range in the TEMPO Extension Japan trial: ALT, 5-45 U/L; AST, 10-40 U/L.

Abbreviations: ALT, alanine aminotransferase; AST, aspartate aminotransferase; TEMPO, Tolvaptan Efficacy and Safety in Management of Autosomal Dominant Polycystic Kidney Disease and its Outcomes.

A

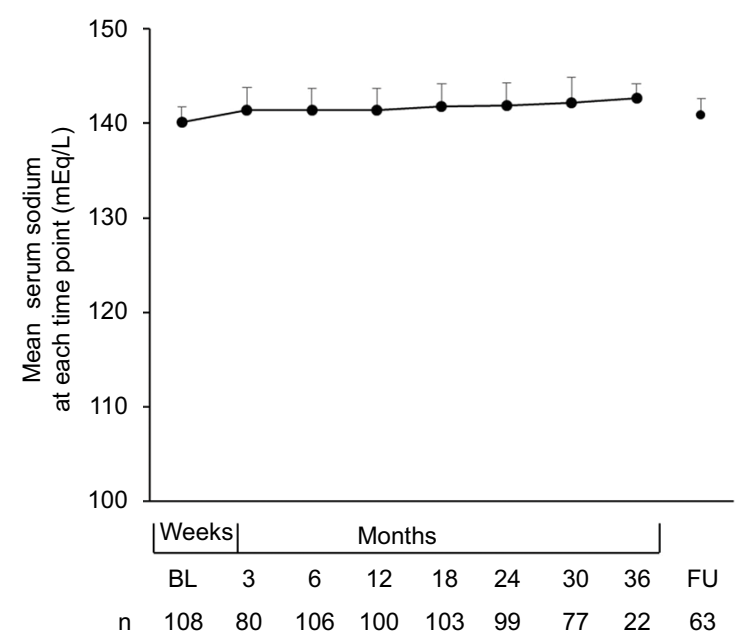

B

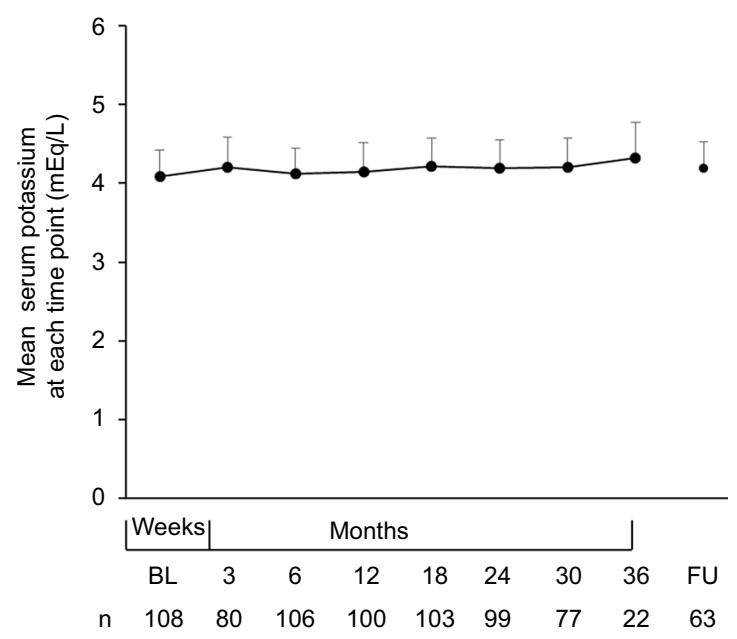

Figure 5 Serum sodium and potassium levels at each time point.

Notes: Serum $(\mathbf{A})$ sodium and $(\mathbf{B})$ potassium levels. Data are expressed as mean \pm SD.

Abbreviations: BL, baseline; FU, follow-up; n, number of patients.

tolvaptan, $\mathrm{n}=484$ placebo) randomized in the TEMPO 3:4 trial, 948 (excluding Japan) completed the trial and 871 of them elected to enroll in TEMPO 4:4 ( $n=557$, early-treatment group, $58.0 \%$ of those on prior tolvaptan; $n=314$, delayed-treatment group, $64.9 \%$ of those on prior placebo). In general, the safety profile for tolvaptan in TEMPO 4:4 was similar to that of the tolvaptan arm of TEMPO 3:4. Briefly, most AEs were related to the aquaretic effect of tolvaptan. The incidence of hepatic 

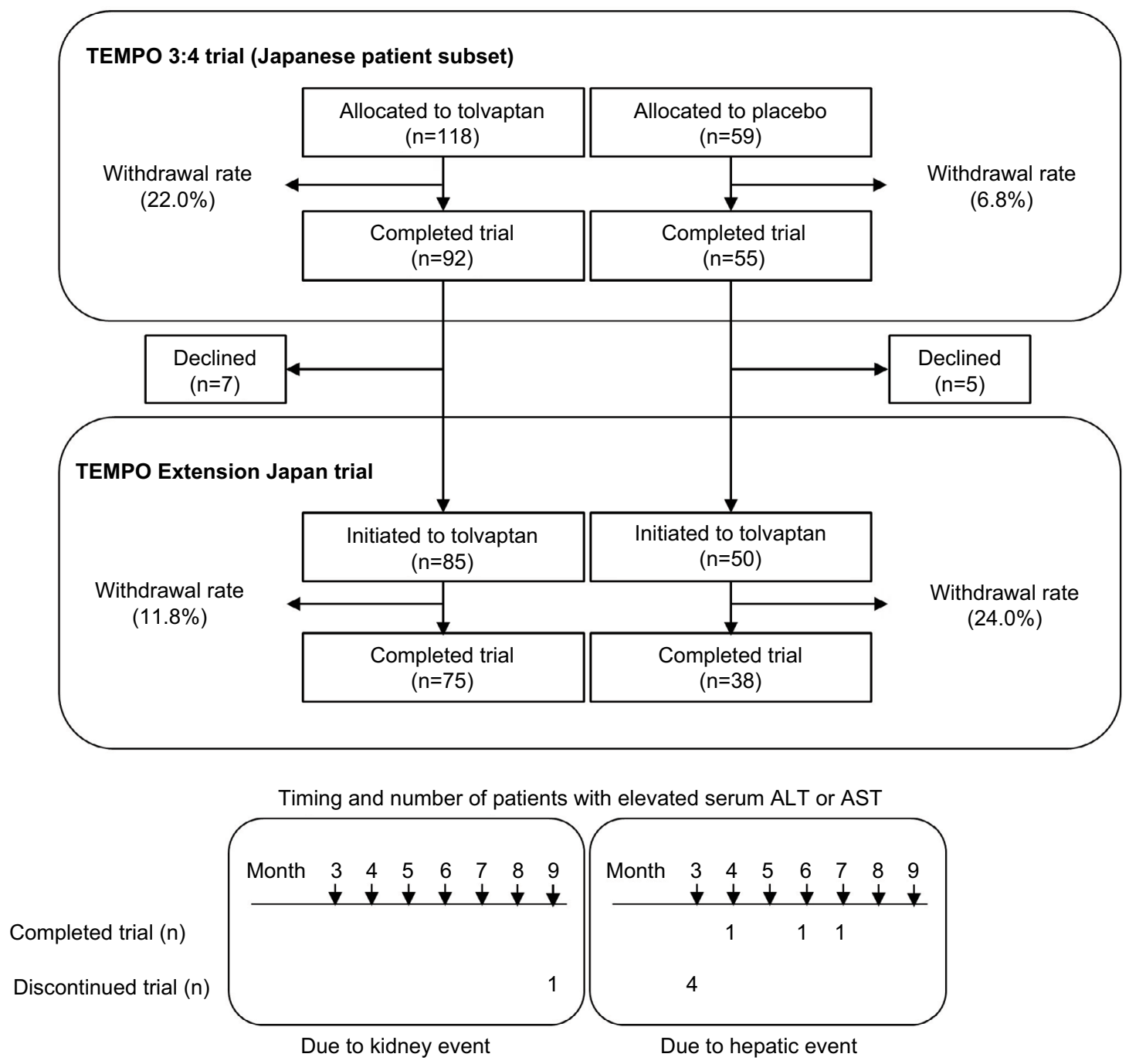

Figure 6 Summary of the TEMPO Extension Japan Trial after the TEMPO 3:4 trial. ${ }^{7.8}$

Abbreviation: TEMPO, Tolvaptan Efficacy and Safety in Management of Autosomal Dominant Polycystic Kidney Disease and its Outcomes.

events in the delayed-treatment patients was similar to that observed in the tolvaptan-treated patients in TEMPO 3:4. One patient in the delayed-treatment group met the criterion for Hy's Law and this has been previously reported. ${ }^{11}$

\section{Conclusion}

ADRs observed in this extension trial were similar to those identified in the TEMPO 3:4 trial, and hepatic events were not progressive. During the first 3 years of tolvaptan use, regular monitoring of serum ALT, AST, and T-Bil levels is important to reduce the risk of serious hepatic events. However, further discussion is required regarding the frequency of the monitoring, especially after 3 years of tolvaptan use.

\section{Acknowledgments}

Data described in the article were all provided by the Department of Clinical Development (Otsuka Pharmaceutical Co,
Ltd, Osaka, Japan) to the Department of Medical Affairs (Otsuka Pharmaceutical Co, Ltd, Tokyo, Japan). The authors' sincere thanks are due to Jun-ichi Hashimoto, Kyoji Imaoka, and Taku Seriu - for approval of this project and generous support; to Mika Nakanishi, Yuko Yamashige, Emi Tomotake, Kei Hayashi, Hiroki Inamura, and Saki Misono - for implementation of the TEMPO Extension Japan trial; to Kazumasa Kishi, Kentaro Ouchi, Eileen Liew, Yoshiyuki Shibasaki, and Sayaka Tachikawa - for critical revision of the manuscript; and to Hisashi Nagamoto and Kenji Uehara for quality control of this manuscript. The present trial was funded by Otsuka Pharmaceutical Co, Ltd, Tokyo, Japan.

In addition to the authors, Eiji Higashihara (Kyorin University School of Medicine, Tokyo, Japan) contributed as a medical advisor for the TEMPO Extension Japan trial and, the following investigators participated in this study: Akio Matsubara (Graduate School of Biomedical and Health 
Sciences, Hiroshima University, Hiroshima), Eiji Ishimura (Osaka City University Graduate School of Medicine, Osaka), Hajime Hasegawa (Saitama Medical Center, Saitama Medical University, Saitama), Hideo Yasuda (Hamamatsu University School of Medicine, Shizuoka), Ichiei Narita (Niigata University Graduate School of Medical and Dental Sciences, Niigata), Kazuhiko Tsuruya (Graduate School of Medical Sciences, Kyushu University, Fukuoka), Kazunari Yoshida (Kitasato University School of Medicine, Tokyo), Keitaro Yokoyama (Jikei University School of Medicine, Tokyo), Kenmei Takaichi (Toranomon Hospital, Tokyo), Kikuo Nutahara (Kyorin University School of Medicine, Tokyo), Koichi Asahi (Fukushima Medical University, Fukushima), Koichi Kamura (National Hospital Organization Chiba-East Hospital, Chiba), Koichi Seta (National Hospital Organization Kyoto Medical Center, Kyoto), Kosaku Nitta (Tokyo Women's Medical University, Tokyo), Kouju Kamata (Kitasato University School of Medicine, Kanagawa), Masayuki Endo (Tokai University School of Medicine, Kanagawa), Michio Kuwahara (Shuuwa General Hospital, Saitama), Mikio Okamura (Ohno Memorial Hospital, Osaka), Naoki Nihei (Chiba University Graduate School of Medicine, Chiba), Sadayoshi Ito (Tohoku University Graduate School of Medicine, Miyagi), Saori Nishio (Hokkaido University Graduate School of Medicine, Hokkaido), Shigeaki Muto (Jichi Medical University, Tochigi), Shinichi Uchida (Graduate School of Medical and Dental Sciences, Tokyo Medical and Dental University, Tokyo), Shuichi Tsuruoka (Nippon Medical School, Tokyo), Takeshi Matsubara (Kyoto University Graduate School of Medicine, Kyoto), Taku Miyoshi (Kumamoto University Graduate School of Medical Sciences, Kumamoto), Yoshifumi Ubara (Toranomon Hospital, Tokyo), Yoshitaka Isaka (Osaka University Graduate School of Medicine, Osaka), and Yukio Yuzawa (School of Medicine, Fujita Health University, Aichi).

\section{Author contributions}

SM, SH, TO, and MY contributed to implementation of the TEMPO Extension Japan trial and critically revised the manuscript. KN and HT contributed to interpretation of the data and writing of the first draft of the manuscript. Editorial assistance in this study was provided by the Department of Clinical Development and Department of Medical Affairs, Otsuka Pharmaceutical Co, Ltd, Osaka, Japan. The submitted manuscript has been approved by all the authors.

\section{Disclosure}

SH has received honoraria for presentations, paid travels, a payment for writing an expert report, and research funding from Otsuka Pharmaceutical; in addition, he has been a medical advisor of the TEMPO Extension Japan trial. SM has received honoraria for presentations, paid travel, and research funding from Otsuka Pharmaceutical. TO, MY, HT, and $\mathrm{KN}$ are employees of Otsuka. SM and SH belong to an endowed department sponsored by Otsuka Pharmaceutical Co, Ltd. The authors report no other conflicts of interest in this work.

\section{References}

1. Grantham JJ, Torres VE, Chapman AB, et al; CRISP Investigators. Volume progression in polycystic kidney disease. $N$ Engl J Med. 2006;354(20):2122-2130.

2. Higashihara E, Nutahara K, Kojima M, et al. Prevalence and renal prognosis of diagnosed autosomal dominant polycystic kidney disease in Japan. Nephron. 1998;80(4):421-427.

3. Kistler AD, Poster D, Krauer F, et al. Increases in kidney volume in autosomal dominant polycystic kidney disease can be detected within 6 months. Kidney Int. 2009;75(2):235-241.

4. Gattone VH, Wang X, Harris PC, Torres VE. Inhibition of renal cystic disease development and progression by a vasopressin V2 receptor antagonist. Nat Med. 2003;9(10):1323-1326.

5. Torres VE, Wang X, Qian Q, Somlo S, Harris PC, Gattone VH 2nd. Effective treatment of an orthologous model of autosomal dominant polycystic kidney disease. Nat Med. 2004;10(4):363-364.

6. Aihara M, Fujiki H, Mizuguchi H, et al. Tolvaptan delays the onset of end-stage renal disease in a polycystic kidney disease model by suppressing increases in kidney volume and renal injury. J Pharmacol Exp Ther. 2014;349(2):258-267.

7. Torres VE, Chapman AB, Devuyst O, et al; TEMPO 3:4 Trial Investigators. Tolvaptan in patients with autosomal dominant polycystic kidney disease. N Engl J Med. 2012;367(25):2407-2418.

8. Muto S, Kawano H, Higashihara E, et al. The effect of tolvaptan on autosomal dominant polycystic kidney disease patients: a subgroup analysis of the Japanese patient subset from TEMPO 3:4 trial. Clin Exp Nephrol. 2015;19(5):867-877.

9. Temple R. Hy's law: predicting serious hepatotoxicity. Pharmacoepidemiol Drug Saf. 2006;15:241-243.

10. Torres VE, Chapman AB, Devuyst O, et al; TEMPO 4:4 Trial Investigators. Multicenter, open-label, extension trial to evaluate the long-term efficacy and safety of early versus delayed treatment with tolvaptan in autosomal dominant polycystic kidney disease: the TEMPO 4:4 Trial. Nephrol Dial Transplant. Epub 2017 Mar 31:1-13.

11. Watkins PB, Lewis JH, Kaplowitz N, et al. Clinical pattern of tolvaptan-associated liver injury in subjects with autosomal dominant polycystic kidney disease: analysis of clinical trials database. Drug Saf. 2015;38(11):1103-1113.

12. Slizgi JR, Lu Y, Brouwer KR, et al. Inhibition of human hepatic bile acid transporters by tolvaptan and metabolites: contributing factors to drug-induced liver injury. Toxicol Sci. 2016;149:237-250.

13. Kinugawa $K$, Sato N, Inomata T, Shimakawa T, Iwatake N, Mizuguchi $\mathrm{K}$. Efficacy and safety of tolvaptan in heart failure patients with volume overload. Circ J. 2014;78(4):844-852. 


\section{Supplementary material}

Table SI Full list of trial sites and IRB approval dates

\begin{tabular}{|c|c|c|c|}
\hline $\begin{array}{l}\text { Trial site } \\
\text { number }\end{array}$ & Trial site and address & Principal investigator & $\begin{array}{l}\text { IRB approval } \\
\text { date, year/ } \\
\text { month/day }\end{array}$ \\
\hline $\mathrm{I}$ & Hokkaido University Hospital, Kita I4, Nishi 5, Kita, Sapporo, Hokkaido 060-8648 & Saori Nishio, Sekiya Shibazaki & $2010 / 11 / 16$ \\
\hline 2 & Teikyo University Hospital, 2-I I-I Kaga, Itabashi, Tokyo I73-8605 & Shigeo Horie, Satoru Muto & $2010 / 11 / 16$ \\
\hline 3 & Nippon Medical School Hospital, I-I-5 Sendagi, Bunkyo, Tokyo I I 3-8603 & $\begin{array}{l}\text { Yasuhiko lino, Shuichi } \\
\text { Tsuruoka }\end{array}$ & $2010 / 10 / 28$ \\
\hline 4 & Kyorin University Hospital, 6-20-2 Shinkawa, Mitaka, Tokyo I8I-86II & Kikuo Nutahara & $2010 / 12 / 8$ \\
\hline 5 & $\begin{array}{l}\text { Kitasato University Hospital, I-I5-I Kitazato, Minami, Sagamihara, Kanagawa 252- } \\
0375\end{array}$ & Koju Kamata & $2010 / 11 / 17$ \\
\hline 6 & $\begin{array}{l}\text { Kitasato University Hospital, I-I5-I Kitazato, Minami, Sagamihara, Kanagawa 252- } \\
0375\end{array}$ & Kazunari Yoshida & $2010 / 11 / 17$ \\
\hline 7 & $\begin{array}{l}\text { Niigata University Medical and Dental Hospital, I-754, Asahimachidori, Chuo, } \\
\text { Niigata, Niigata } 95 \text { I-8520 }\end{array}$ & Ichiei Narita & $2010 / 10 / 26$ \\
\hline 8 & Osaka University Hospital, 2-15 Yamadaoka, Suita, Osaka 565-087I & Yoshitaka Isaka & $2010 / 12 / 13$ \\
\hline 9 & Tohoku University Hospital, I-I, Seiryo-cho, Aoba-ku, Sendai, Miyagi 980-8574 & Sadayoshi Ito & $2010 / 12 / 6$ \\
\hline 10 & Jichi Medical School Hospital, 33 I I-I Yakushiji, Shimotsuke, Tochigi 329-0498 & Eiji Kusano, Shigeaki Muto & $2011 / 2 / 25$ \\
\hline II & Saitama Medical Center, 198I, Kamoda, Kawagoe, Saitama 350-8550 & Hajime Hasegawa & $2011 / 2 / 17$ \\
\hline 12 & Chiba University Hospital, I-8-I, Inohana, Chuo, Chiba, Chiba 260-8677 & Naoki Nihei & $2010 / 11 / 15$ \\
\hline 13 & Toranomon Hospital, 2-2-2 Toranomon, Minato, Tokyo I05-8470 & Kenmei Takaichi & $2011 / 2 / 25$ \\
\hline 14 & The Jikei University Hospital, 3-19-18 Nishishinbashi, Minato, Tokyo & $\begin{array}{l}\text { Tatsuo Hosoya, Keitaro } \\
\text { Yokoyama }\end{array}$ & $2011 / 9 / 27$ \\
\hline 15 & Tokai University Hospital, I43 Shimokasuya, Isehara, Kanagawa 259-II 93 & Masayuki Endoh & $2010 / 12 / 22$ \\
\hline 16 & $\begin{array}{l}\text { Toranomon Hospital Kajigaya, I-3-I Kajigaya, Takatsu, Kawasaki, Kanagawa 213- } \\
8587\end{array}$ & Yoshifumi Ubara & $2011 / 1 / 11$ \\
\hline 17 & Ohno Memorial Hospital, I-26-10 Minamihorie, Nishi, Osaka, Osaka 550-00I5 & Mikio Okamura & $2011 / 1 / 27$ \\
\hline 18 & $\begin{array}{l}\text { Hiroshima University Hospital, I-2-3 Kasumi, Minami, Hiroshima, Hiroshima 734- } \\
855 \text { I }\end{array}$ & Akio Matsubara & $2011 / 5 / 9$ \\
\hline 19 & $\begin{array}{l}\text { Fukushima Medical University, Hospital, I Hikarigaoka, Fukushima, Fukushima } \\
960-1295\end{array}$ & Koichi Asahi & $2011 / 3 / 2$ \\
\hline 20 & $\begin{array}{l}\text { National Hospital Organization, Chiba-East Hospital, } 673 \text { Nitona, Chuo, Chiba, } \\
\text { Chiba } 260-8712\end{array}$ & Koichi Kamura & $2011 / 4 / 26$ \\
\hline 21 & Shuwa General Hospital, I200, Yaharashinden, Kasukabe Saitama 344-0035 & Michio Kuwahara & $2011 / 4 / I I$ \\
\hline 22 & $\begin{array}{l}\text { Tokyo Women's Medical, University Hospital, 8-I Kawada, Shinjuku, Tokyo I62- } \\
8666\end{array}$ & Kosaku Nitta & $20114 / 27$ \\
\hline 23 & Osaka City University Hospital, I-5-7 Asahimachi, Abeno, Osaka, Osaka 545-8586 & Eiji Ishimura & $2011 / 3 / 23$ \\
\hline 24 & Kyoto University Hospital, 54, Kawara, Shogoin, Sakyo, Kyoto, Kyoto 606-8507 & $\begin{array}{l}\text { Atsushi Fukatsu, Noriyuki } \\
\text { lehara, Takeshi Matsubara }\end{array}$ & $2011 / 9 / 12$ \\
\hline 25 & Kyushu University Hospital, 3-I-I, Maidashi, Higashi, Fukuoka, Fukuoka 8I2-8582 & Kazuhiko Tsuruya & $2011 / 2 / 24$ \\
\hline 26 & $\begin{array}{l}\text { Hamamatsu University School of Medicine, University Hospital, I-20-I Handayama, } \\
\text { Higashi, Hamamatsu, Shizuoka 43I-3192 }\end{array}$ & $\begin{array}{l}\text { Yoshihide Fujigaki, Hideo } \\
\text { Yasuda }\end{array}$ & $2011 / 4 / 14$ \\
\hline 27 & $\begin{array}{l}\text { National Hospital Organization, Kyoto Medical Center, I-I, Mukaihata, Fukakusa, } \\
\text { Fushimi, Kyoto 612-8555 }\end{array}$ & Koichi Seta & $2011 / 5 / 25$ \\
\hline 28 & $\begin{array}{l}\text { Fujita Health University Hospital, I-98 Dengakugakubo, Kutsukake, Toyoake, Aichi } \\
470-1192\end{array}$ & Yukio Yuzawa & $2011 / 4 / 27$ \\
\hline 29 & $\begin{array}{l}\text { Kumamoto University Hospital, I-I-I Honjyo, Chuo, Kumamoto, Kumamoto } \\
860-8556\end{array}$ & Taku Miyoshi & $2011 / 5 / 23$ \\
\hline 30 & $\begin{array}{l}\text { Tokyo Medical and Dental University Hospital Faculty of Medicine, I-5-45 Yushima, } \\
\text { Bunkyo, Tokyo I I3-85 I } 9\end{array}$ & Sei Sasaki, Shinichi Uchida & $2011 / 7 / 25$ \\
\hline
\end{tabular}

Abbreviation: IRB, institutional review board. 


\section{Publish your work in this journal}

Drug, Healthcare and Patient Safety is an international, peer-reviewed open access journal exploring patient safety issues in the healthcare continuum from diagnostic and screening interventions through to treatment, drug therapy and surgery. The journal is characterized by the rapid reporting of reviews, original research, clinical, epidemiological and post-marketing surveillance studies, risk management, health literacy and educational programs across all areas of healthcare delivery. The manuscript management system is completely online and includes a very quick and fair peer-review system. Visit http://www.dovepress.com/ testimonials.php to read real quotes from published authors.

Submit your manuscript here: https://www.dovepress.com/drug-healthcare-and-patient-safety-journal 